\title{
Beckenbodenprobleme - auch bei Zustand nach Sectio
}

\author{
Susanne Schwärzler, Beckenbodentrainerin, Kempten
}

\section{Auch nach einem Kaiserschnitt können Beckenbodenprobleme auftreten. Eine erfahrene Beckenbodentrainerin beschreibt, wie man die Beckenbodenschwäche dieser Frauen er- kennen kann und welche Übungen in der Rückbildungsgymnastik für Frauen mit Zustand nach Sectio besonders empfehlenswert sind.}

Der Beckenboden ist ein „Geburtsorgan“. Fast alle Menschen sind in ihm gezeugt, ausgetragen und durch ihn geboren worden. Ein bewusster Umgang mit ihm macht stark und einfühlsam nicht nur für die Geburt, sondern auch in der Sexualität, während der Schwangerschaft und im ganz normalen Alltag (Beruf und Sport). Er ist der „Grund“ und „der innere Halt“ unseres Beckens.

Als Basis trägt und balanciert er unseren Oberkörper mit Kopf und Armen sowie die Beine und Füße. Ohne „Kraft von innen“" werden die Belastungen dagegen an die Gelenke und Bandscheiben abgegeben. Dazu passt die Beobachtung, dass afrikanische Frauen, die viele Lasten auf dem Kopf transportieren und dabei automatisch ihren Beckenboden anspannen, trotz zahlreicher Geburten eher selten Senkungsbeschwerden haben.

Der Beckenboden steht mit der Bauchmuskulatur und den umliegenden Beckenmuskeln und Fazien in Beziehung. Vor allem die mittlere und innere Beckenbodenschicht arbeitet stark mit der Bauchmuskulatur zusammen. Natürlich ist dann auch die Rücken-, Gesäß-, Hüftbeuger- und Oberschenkelmuskulatur mitbetroffen.

Ein Kaiserschnitt hat im Vergleich zu einer Spontangeburt keinen direkten Einfluss auf den Beckenboden. Der Beckenboden wird ja umgangen. Oft sind es aber gerade diese Frauen, die im Rückbildungsgymnastikkurs wenig Zugang zu ihrem Beckenboden finden, da sie ihn nicht „erlebt“ haben. Ich kenne viele Frauen, die wenige Tage oder Wochen nach einer Sectio über Rückenbeschwerden klagen. Kein Wunder: Bei der Durchtrennung der Bauchmuskulatur (vor allem der querverlaufenden Muskeln) wird die Verbindung zum Becken instabil. Alle Muskeln, die am Becken befestigt sind, leiden unter dieser Instabilität.

Auch die Sitzbeinhöcker sind betroffen. Sie können ihre Querspannung (über den M. transversus perinei profundus und M. transversus perinei superficialis) ohne die Mithilfe der querverlaufenden (M. transversus abdominis) und schrägen Bauchmuskulatur (M. obliquus abdominis) nicht mehr halten und öffnen sich. Dabei kippt das Becken nach vorne, eine Hohlkreuzhaltung entsteht! Die Rückenmuskulatur ist dann überfordert.

Auch die Beine haben über den „lockeren“ $M$. piriformis und den M. obturatorius internus sowie den $M$. coccygeus weniger Spannung. Daraus resultiert eine X-Beinhaltung. Knie- und Fußgelenke bekommen die Belastung und den Druck ab.

Ein jahrelanger, schwacher Beckenboden und ein fehlendes Beckenbodenbewusstsein im Alltag führen auf die
Dauer zu einer Beckenbodenschwäche. Haltungsschwäche, Senkung, Inkontinenz, Gelenk- und Bandscheibenprobleme sind die Folgen.

Als Beckenbodentrainerin unterrichtete ich seit über 20 Jahren junge Mütter und ältere Betroffene mit Beckenbodenschwäche (Senkung, Inkontinenz). Dazu habe ich ein speziell entwickeltes Konzept mit einem systematischen und schonenden Aufbautraining bei Beckenbodenschwäche entwickelt. Verschiedene Übungen in bestimmten Ausgangslagen (z.B. Rücken-, Bauch- und Seitenlage) sowie das Einbeziehen der Fußstellungen (fersenbetont oder zehenspitzenbetont) unterstützen mechanisch und reflektorisch die Beckenbodenmuskulatur und die Zusammenarbeit mit der Bauchmuskulatur.

Auffallend ist bei Frauen mit Bauchnarben (Z. n. Sectio oder Bauchoperation) ein Abkippen des Beckens bei Belastung in fast allen Körperlagen. Das Zusammenspiel der Bauch- und Beckenbodenmuskulatur muss erst wieder antrainiert werden.

\section{Eine Beckenboden- schwäche erkennen}

Mit der folgenden einfachen Übung kann die Leiterin eines Rückbildungs- 


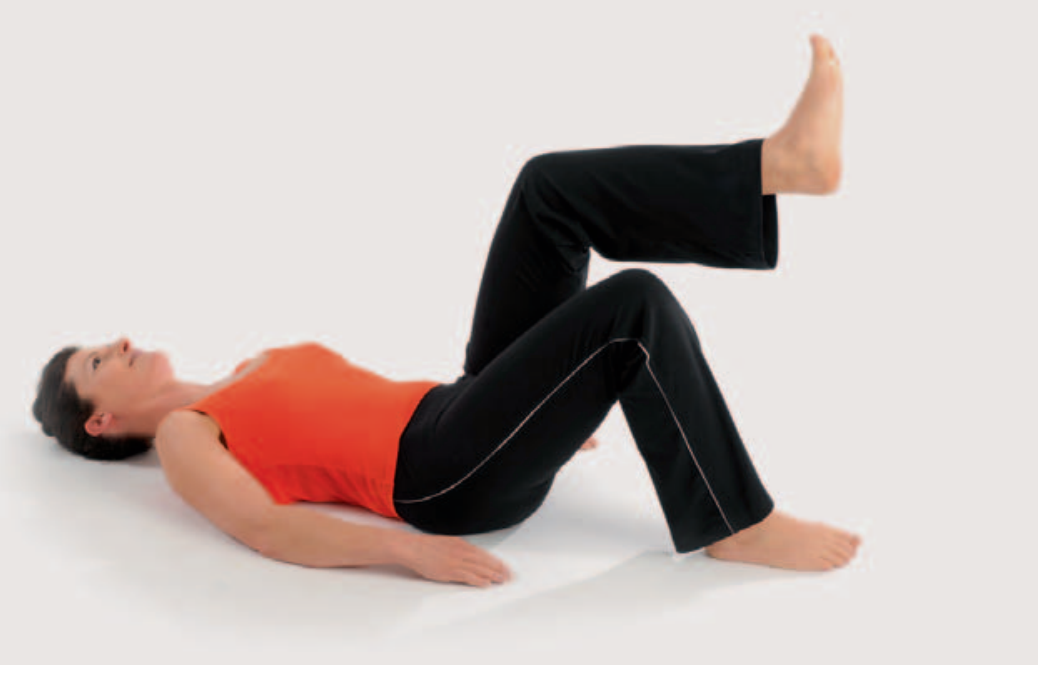

Abb. 1: Übung 1: Beckenschaukel mit Anheben eines Beines.

gymnastikkurses bei Frauen mit Z. n. Sectio oder einer anderen Bauchoperation eine Beckenboden- oder Bauchmuskelschwäche erkennen:

\section{Übung 1: Beckenschaukel mit Anheben eines Beines}

\section{Übungsablauf während der Ausatmung:}

- Durch den leicht geöffneten Mund ausatmen

- Zuerst die Schließmuskeln aktivieren

- Die Sitzbeinhöcker zueinander ziehen

- Dabei das große Becken zur Unterlage kippen (Schambein zieht zum Kinn)

- Den „inneren Aufzug“ nach innen oben ziehen

- Zuletzt das angewinkelte Bein von der Unterlage anheben.

\section{Wahrnehmung:}

- Das Lungenvolumen verkleinert sich

- Die Bauchdecke spannt sich an

- Der untere Rücken streckt sich

- Alle Beckenbodenschichten sind angespannt

- Anspannung in der unteren, schrägen Bauchmuskulatur

Bei dieser Übung ist bei Sectio-Frauen bereits ein seitliches Abkippen des
Beckens erkennbar. Die Bauchmuskulatur kann der Belastung nicht standhalten. Bauchmuskulatur und Beckenboden können nicht zusammenarbeiten. Der Zugang zum Beckenboden ist nicht gegeben. Das Becken kippt seitlich ab.

\section{Übungen für Frauen mit Z. n. Sectio}

Die folgenden Übungen sind beispielhafte Basisübungen für SectioFrauen im Rückbildungsgymnastikkurs. Sie stärken die Bauchmuskulatur. Dadurch lässt sich das Zusammenspiel mit der Beckenbodenmuskulatur deutlich verbessern.

Bei jeder dieser Übungen kann diagonal ein Bein oder die Ferse angehoben werden, um die Wirkung der Übung zu verstärken.

\section{Das Becken darf dabei nicht seit-} lich abkippen!

Wenn die Frau bemerkt, dass ihr Becken abkippt, darf sie nur eine Ferse anheben (nicht das ganze Bein), um das Becken stabil zu halten.
Tipps für Kursleiterinnen:

- Sprachtraining: Die Aussprache des Vokals „u“ aktiviert die innere Beckenbodenspannung.

- Fußstellung: Beim Einbeziehen der Zehenspitzen- oder Fersenstellung vor Beginn der Übung kann die „Schließmuskelschicht“ des Beckenbodens besser wahrgenommen, aktiviert und angehalten werden.

\section{Übung 2:}

\section{Ausgangsstellung Stehen}

Diese Übung entspricht der „Beckenschaukel“ im Stehen. Die Ausgangsstellung Stehen sollte von den Frauen auch im Alltag zum Training und zum Schutz vor einer Beckenbodenbelastung angewandt werden, z. B. beim Stehen an der Küchenzeile, in der Warteschlange, an der Ampel, beim Tragen des Kindes, beim Telefonieren oder Zähneputzen.

Als Hilfsmodell kann man sich einen verlängerten Schwanz vorstellen, auf den man sich absetzt. Die Knie sollten bei dieser Übung nicht über den Mittelfuß hinaus vorgeschoben werden.

\section{Übungszeit: 2 Minuten}

\section{Übungsziele:}

- An- und Entspannung des Beckenbodens

- Wahrnehmen des Zusammenspiels zwischen Beckenboden und Bauch-, Rücken-, Gesäß-, Schulter- und Beinmuskulatur

\section{Ausgangsposition}

- Aufrechtes Stehen

- Die Beine stehen hüftbreit parallel

- Eine Hand liegt auf dem Bauch, die andere auf dem Rücken im LWSBereich

Übungsablauf während der Einatmung

- Tief durch die Nase einatmen

- Das große Becken nach vorne kippen (Steißbein zeigt nach hinten)

- Alle Beckenbodenschichten locker lassen 


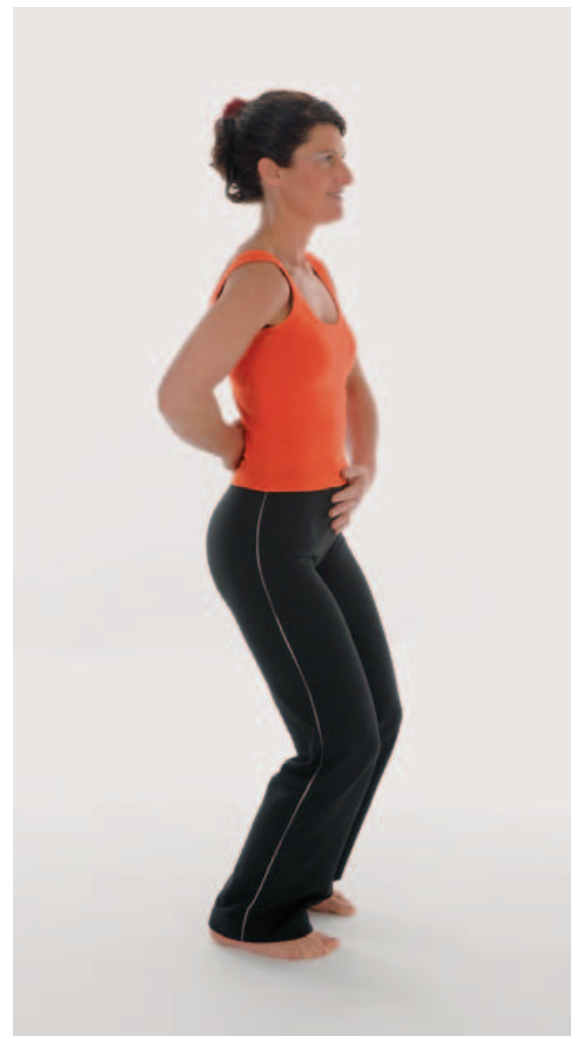

Abb. 2: Übung 2: Ausgangsstellung Stehen.

\section{Wahrnehmung}

- Die Körperhaltung ist entspannt

- Der Beckenboden ist entspannt

- Der Unterbauch ist entspannt (wölbt sich nach vorne)

- Das Becken ist weit (Steißbein zeigt nach hinten)

- Eine leichte Hohlkreuzhaltung entsteht

\section{Übungsablauf während der \\ Ausatmung}

- Tief durch den leicht geöffneten Mund ausatmen

- Dabei nach einander die Schließmuskeln aktivieren

- Die Sitzbeinhöcker zusammenziehen

- Das große Becken nach hinten kippen (Steißbein nach vorne ziehen)

- Den „inneren Aufzug“ in Richtung Bauchraum ziehen

\section{Wahrnehmung}

- Das kleine Becken kippt nach oben (Steißbein zieht zum Kinn)
- Der untere Rücken streckt sich

- Beinspannung entsteht

- Alle Beckenbodenschichten sind angespannt

- Die Knie sind über den Zehenspitzen

- Die Körperhaltung ist angespannt

\section{Übung 3: Ausgangsstellung Seitenlage}

Übungszeit: 2 Minuten

\section{Übungsziele:}

- Wahrnehmung der An- und Entspannung der Beckenbodenmuskulatur

- Kräftigung der unteren Bauch-, Rücken-, Gesäß- und Beinmuskulatur

- Beckenbodenanspannung

\section{Ausgangsposition}

- Liegen in Seitenlage

- Angewinkelte Beine

- Der Kopf liegt auf dem angewinkelten Unterarm

- Der andere Arm stützt sich vor dem Oberkörper auf

- Fersen, Gesäß, Schultern und Kopf bilden eine Linie

\section{Übungsablauf während der Einatmung}

- Tief durch die Nase einatmen

- Das große Becken bewegt sich nach vorne (Steißbein nach hinten)

\section{Wahrnehmung}

- Das Lungenvolumen vergrößert sich

- Entspannte, vorgewölbte Bauchdecke

- Eine leichte Hohlkreuzhaltung entsteht

- Alle Beckenbodenschichten sind entspannt

- Das Becken ist weit

- Die Taille liegt entspannt auf der Unterlage

- Die Beine sind locker

\section{Übungsablauf während der Ausatmung}

- Durch den leicht geöffneten Mund ausatmen

- Zuerst die Schließmuskeln aktivieren

- Die Sitzbeinhöcker zueinander ziehen

- Zuletzt den „inneren Aufzug“ nach innen oben ziehen

\section{Wahrnehmung}

- Das Lungenvolumen verkleinert sich

- Das große Becken kippt nach hinten (Schambein zieht zum Kinn)

- Die Bauchdecke spannt sich an

- Der untere Rücken streckt sich

- Alle Beckenbodenschichten sind angespannt

- Die Taille hebt sich von der Unterlage ab

- Gesamtkörperspannung

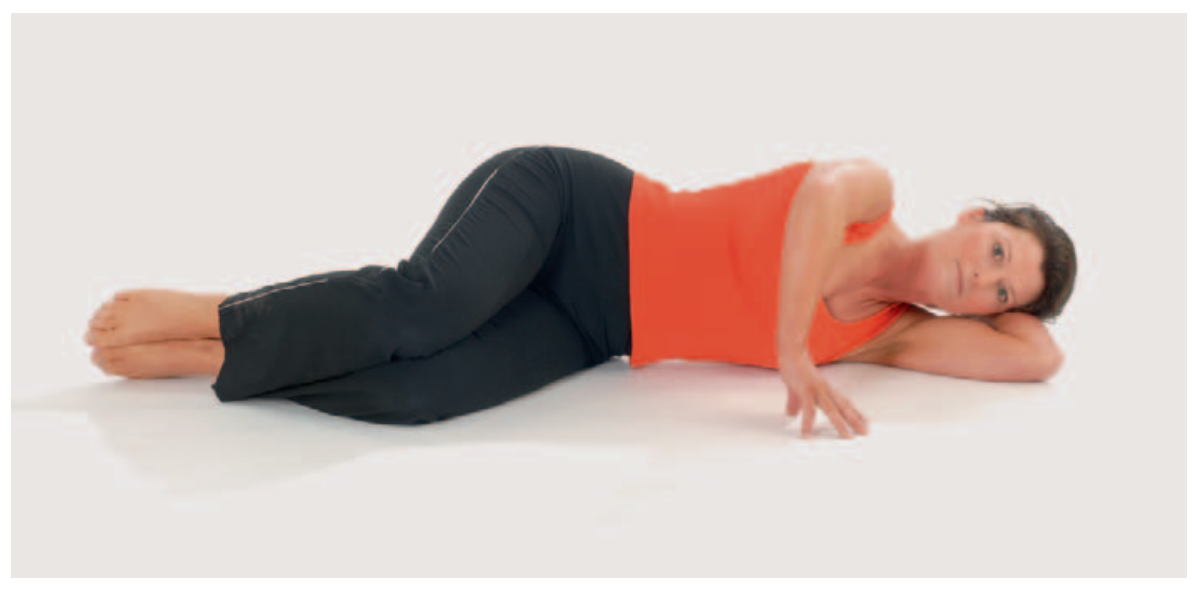

Abb. 3: Übung 3: Ausgangsstellung Seitenlage. 


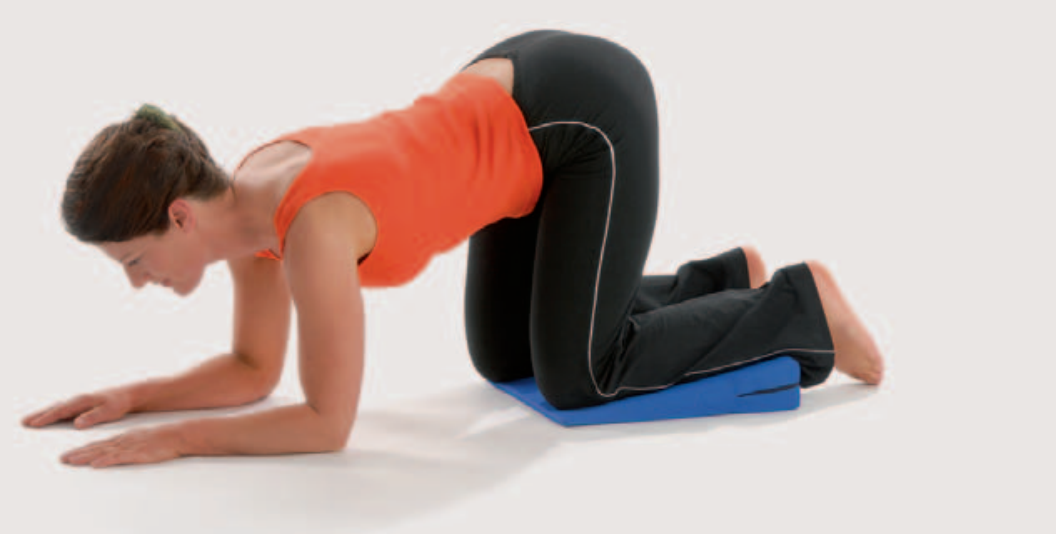

Abb. 4: Übung 4: Ausgangsstellung Knie-Ellenbogen-Lage.

\section{Übung 4: Ausgangsstellung Knie-Ellenbogen-Lage}

Übungszeit: 2 Minuten

\section{Übungsziele:}

- Entspannung und Entlastung des Beckenbodens

- Kräftigung der Bauchmuskulatur

\section{Ausgangsposition}

- Vierfüßlerstand, von dort wird das Gewicht auf die Ellenbogen verlagert

- Die Knie befinden sich unter dem Becken

- Die Ellenbogen sind unter den Schultern

- Kopf, Schultern und Gesäß bilden eine Ebene

- Ein untergelegtes Kissen unterstützt die Entlastungsübung

\section{Übungsablauf während der Einatmung}

- Tief durch die Nase einatmen

- Das kleine Becken nach hinten kippen (Steißbein zeigt nach hinten oben)

\section{Wahrnehmung}

- Hochkreuzhaltung entsteht

- Alle Beckenbodenschichten sind entspannt

\section{Buchtipp}

Susanne Schwärzler:

Hippokrates Verlag 2011

\section{6 Übungseinheiten}

- Zuletzt den „inneren Aufzug“ nach innen oben ziehen

\section{Wahrnehmung}

- Der untere Rücken streckt sich

- Die Bauchdecke spannt sich an

- Alle Beckenbodenschichten sind angespannt, aber druckentlastet

Diese Übungen (10-20-mal das Becken vor- und zurückkippen) sollten von den Frauen auch Zuhause mehrmals täglich durchgeführt werden.

Im Rückbildungsgymnastikkurs kann man immer wieder eine kleine Übungseinheit (mindestens 5-mal das Becken kippen) einbauen, um den Trainingserfolg zu sichern.

\section{Anschrift der Autorin:}

Susanne Schwärzler

Dottenried 44

87439 Kempten

Beckenbodentraining im Rückbildungsgymnastikkurs

\section{Beckenbodentraining im Rückbildungsgymnastikkurs}

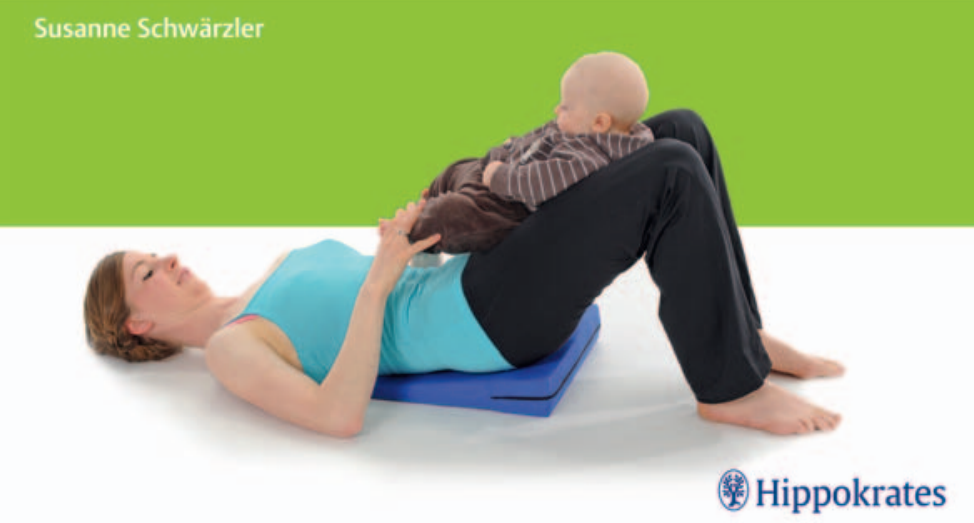

